The papers given at the conference (which will be published in the Proceedings of the Society) may be regarded as a continuation of earlier symposia on Colonial problems ${ }^{4}$ and on nutritional conditions in prisoner-of-war camps in Asia ${ }^{5}$. They should serve a useful purpose in providing information regarding the actual and potential food supplies of under-developed areas, and in this way should assist in furthering the work being carried out on these topics under the auspices of the Colonial Office and other national and international agencies.

Francis AyLward

1 Sorre, M., "Les Fondements de la Geographie Humaine", I (Paris, 1947).

"De Castro, J., “Geography of Hunger”, 36 (Iondon: Victor Gol,

Nature, 168, 1100 (1951).

- Symposium, Proc. Nutrit. Soc., 5, 1 (1946).

symposium, Proc. Nutrit. Soc., 5, 107 (1946).

\section{APPLICATIONS OF COMMUNICATION THEORY}

$\mathrm{T}$

WO years ago, during September 26-29, 1950, a symposium was held in London of a group of some hundred and twenty men of diverse scientific interests -communication engineers, mathematicians, physicists, biologists, psychologists and others-all of whom were concerned in one way or another with the concept of the communication of information (see Nature, 167,20 ; 1950). The meeting was prompted by the recognition of the importance of recent developments in the theory of communication which revealed, and gave promise of clarifying, connexions between previously largely unrelated fields of research concerned with different aspects of the processes by which living organisms convey information. The purpose of the symposium was to afford the opportunity for a discussion of the nature of this recent work and of its potentialities in various branches of science.

It was decided that there should follow in due course a second symposium having the more limited objective of examining what of practical value had resulted from the application of this theoretical work to the problems of electrical communication, and this second meeting was held in London in the Lecture Theatre of the Institution of Electrical Engineers, during September 22-26. The participants on this occasion were mainly practising communication engineers, though other interests were well represented, and they were even more international than those who attended the earlier symposium. The two hundred and seventy present included ninety visitors from sixteen countries abroad. The chairman of the opening session was Sir Noel Ashbridge, retiring director of technical services of the British Broadcasting Corporation.

In the opening address, Prof. Willis Jackson referred to the two distinct, though closely interrelated, sides of the recent theoretical work : one, the theory of the representation of signals, that is, the analysis of signals into the basic elements-the 'alphabet'-in which it is intended to communicate ; and the other, the statistical theory of communication, that is, the application of probability theory to the communication of these elements. Prof. Jackson remarked on the value of the theory in affording means for the quantitative comparison of different transmission systems (different forms of modulation and coding) ; on the progress being made in the development of techniques for the extraction of signals from a noisy background; and on the considerable activity which it had stimulated in studies such as the analysis and synthesis of speech, the improvement of speech recognition in noisy conditions, the possibilities of compressing the band-width required in speech and television channels, the mechanism of hearing, etc. He remarked that communication theory has perhaps achieved little as yet in the last-named fields, but that to make a reproach of this would be on about the same level as pointing out that Carnot and Kelvin did not invent the gas-turbine. A basic theory is a compass rather than a vehicle, and there is little reason to doubt that, in this case as in others, it will be supported in due course by the inventive imagination on which progress largely depends.

Prof. Jackson then directed attention, in the absence of the author, to the "Summary of Communication Theory" which Dr. D. Gabor had prepared for the benefit of the participants. In this paper Dr. Gabor discussed the parts played, in the development of the theory of communication, by classical Fourier analysis, its modification by treating time as a 'running' variable and the application of probability theory to signal data; and he detailed, in terms based mainly on the work of Dr. C. E. Shannon, the principal concepts, theorems and results concerning the two aspects of the modern work, signal analysis and the statistical theory of communication, mentioned above.

The first two days of the symposium were devoted mainly to problems concerning the detection of signals when mixed with noise, and served to emphasize that the detector must be regarded essentially as giving a probability distribution rather than a precise, error-free, extracted signal. A paper by $\mathrm{P} . \mathrm{H}$. Blundell suggested that it is not permissible to regard the whole of this (a posteriori) distribution, in the mean, as a rate of information, since at any instant during the communication it cannot all be used. This argument aroused considerable discussion which served to clarify some important misconceptions. Prof. Z. Jelonek followed with a summary of the results of a comparative study of various modulation systems in which he had calculated their respective theoretical rates of transmission of information. This paper, and an allied one by Prof. S. Goldman (United States), demonstrated the quantitative value of the statistical theory, and revealed the limited practicable scope which is available for improvement in the methods of modulation now in use.

New contributions to the theory and art of pulse modulation were made by F. de Jager (Netherlands) who described a one-unit code system of pulse-code modulation to which he had given the name "deltamodulation"; by C. W. Earp, who discussed a possible modified form of pulse-code modulation, which he called the "ambiguous index system"; by H. W. F. Groenewout (Netherlands), who gave the results of an investigation of distortion in pulse-code modulation systems ; and by Dr. D. M. MacKay, who raised an interesting problem concerning the transmission of neural impulses.

Allied to these papers were others by R. H. Barker, who dealt with the important problem of the group synchronization of binary digital systems, and by Drs. A. E. Laemmel and D. A. Huffman (United States), who discussed the construction of codes, the former of error-correcting codes involving the 
deliberate inclusion of redundancy, in the form of additional code signals, for the purpose of combating noise, and the latter of codes to remove excessive redundancy, where this exists.

The discussion of the extraction of signals from noise, related more particularly to the detection of pulsed radar signals, and to the comparative efficiency in this respect of alternative methods of detection, was continued in papers by D. E. Hampton, by Dr. W. Meyer-Eppler (Germany), and by Profs. R. M. Fano and D. Middleton (United States). The two latter directed attention to calculation of the a posteriori probability of the received message from the a priori distribution and the assumed statistics of noise, and extended and generalized earlier work on the theory of ideal detectors. Middleton discussed his comparative studies based on several types of optimum test of a statistical hypothesis against an alternative - signal and noise as against noise-- and his consideration of three types of 'observer', namely, the 'NeymanPearson', the 'ideal' (Siegert) and the 'sequential', for which he had computed the corresponding betting-curves. $\mathrm{He}$ also described an analogue electronic device constructed by Dr. R. A. Johnson and himself for measuring the correlation functions of modulated carriers and noise following a non-linear device. One interesting conclusion to be drawn from this series of papers is that no marked inefficiency is associated with the types of detector now in use, and that in fact we cannot hope to go much better, which further illustrates the value of the modern communication theory in enabling calculation of the limits of performance which practical systems cannot hope to exceed. There followed a series of papers by Drs. A. E. Bailey, R. L. Beurle and P. L. Waters, dealing with the question of information storage, and the comparative merits of magnetic, acoustic and electrostatic systems applied to signal integration.

On the morning of the third day the discussion moved from statistical communication theory to a series of papers by D. H. Hamsher (United States), Dr. M. Cotte (France), Prof. K. Küpfmüller (Germany) and Prof. T. Laurent (Sweden), concerned with the transient distortion and delay characteristics of transmission channels and with general signal analysis, chiefly with reference to telegraph and television systems, where the wave-shape of the signals is of prime importance. This was followed during the afternoon by consideration of the information content of pictures and of its transmission by facsimile and television. Papers by Dr. J. Loeb (France), and by Dr. E. C. Cherry and G. G. Gouriet, emphasized the excessive redundancy which exists in facsimile and television signals by virtue of the fact that the probability of a black or grey 'dot' on any real picture is far less than that of a plain surface element. Thus existing practical systems are potentially capable of transmitting patterns which are far more complex than ever arise in real pictures. Dr. Loeb pointed out that scanning is a wasteful and unnecessary procedure with simple pictures, such as sketches, charts, handwriting, etc., and described the principles of pantograph and stopping-spot devices which can trace the picture automatically and the output of which can be coded for transmission to a tracing pencil at the receiver. The second authors proposed a system of velocity modulation applied to television to accomplish the same end. The discussion of these papers centred, somewhat naturally, round the psychological problem of 'recognition'. A succeeding paper by Dr. G. Valensi (Switzerland) dealt with the coding of colour-television signals. He stressed the merits of the modern view of the problem, which regards the black-white version of the picture as one signal and the superposed colour as a second-as distinct from the simultaneous transmission of three pictures, red, blue and green-and proceeded to discuss the possible use of a sub-divided Maxwell colour triangle for defining the colour information.

Speech and hearing formed the subject-matter of the programme on September 25. One group of papers, by J. Berry, D. L. Richards and J. Swaffield, dealt with studies of conversational speech on the telephone. They described statistical measurements of the frequency of occurrence of particular words and syllables, consonants and vowels, strong and weak word-forms, etc., and also an attempt to relate the conventional methods of measuring the performance of speech-links to the concepts of 'channel capacity' and 'information rate' as defined in the modern theory of communication. Another group of papers, by Drs. G. E. Peterson, K. H. Davis, R. Biddulph and S. Balashek (United States) and by Drs. D. Fry and P. Denes, was devoted to the problem of speech recognition and to consideration of the principles whereby a 'machine' might print in standard symbols, or respond in some other recognizable way, to received human speech. These papers emphasized that a great deal of information is lost when speech is transformed to writing and that the main problem is to determine what are the minimal elements of speech which ensure identification. Dr. G. Raisbeck, who presented the paper by Drs. Davis, Biddulph and Balashek, described a machine which is able to identify each of the numerals, one to nine, though only when spoken by the same individual. The problems involved in making it capable of responding similarly to different voices, and of recognizing continuous phrases and sentences, are indeed complex and difficult ones, but carry some far-reaching implications.

These papers were followed by two demonstrations, by W. Lawrence and by Dr. W. G. Tuller (United States), of machines which performed the complementary operation, that of uttering 'spoken' words when driven by electrical stimuli of compara= tively simple form. This fascinating work may provide an important 'synthesis' approach to the study of the speech recognition problem.

A particularly interesting supplementary paper describing a new approach to the theory of hearing was presented by Dr. W. H. Huggins (United States). $\mathrm{H}_{\theta}$ began by considering the measurement of the pitch of a damped wave by means of a 'phase detector' and proceeded to suggest that while the basilar membrane itself does not possess suitable elastic properties to account for the fine pitch discrimination of the human ear, the tectorial membrane, with which it is connected by hair-cells, has similar frequencyselective properties to those of the phase detector. Dr. Huggins showed that these proposals conform well with the experimental results obtained by von Békésy, Galambos and Davis in studies of the cochlea of the cat.

The topics considered on the final day lay somewhat aside the main theme of the symposium but were nevertheless much appreciated by a large audience. Two papers concerned with language and linguistics, presented by D. A. Bell and by Prof. B. Mandelbrot (France), afforded opportunity for a considerable discussion among the linguists present, and revealed how closely the theories of communication and of language are related and how beneficial progress in one can 
be to the other. An important succeeding paper by Dr. Y. Bar Hillel and Prof. R. Carnap (United States) referred to the mis-application by some men of science of the terminology and theorems of statistical communication theory to fields in which the term information is used, presystematically, in a semantic sense (one involving the contents or designata of symbols) or even in a pragmatic sense (one involving the users of these symbols) and to the misleading consequences which can result. They presented the outlines of a theory of semantic information based on the theory of inductive probability recently developed by Prof. Carnap, and suggested that this should serve as a better approximation than the present statistical theory for application to fields like psychology and the social sciences.

An interesting application of the results and methods of communication theory to optics was described by Prof. A. Blanc-Lapierre (France) ; and in a paper entitled "Generators of Information", Dr. D. M. MacKay raised a number of difficult questions, such as - Can we trace the information in a channel back to an ultimate source, or are we restricted to considering information transducers only ? Communication theory generally accepts the a priori existence of an agreed alphabet : where does this arise ?- and illustrated his arguments by descriptions of simple electrical machines.

The symposium concluded with a general discussion, in which Dr. E. C. Cherry gave a summary of the main questions which had been raised during the week, and with an expression of appreciation by Prof. Willis Jackson to the British Broadcasting Corporation and the Ministry of Supply for the financial assistance they had afforded. The proceedings of the symposium are to be published by Butterworths Scientific Publications, Ltd.

\section{THIRD INTERNATIONAL CONGRESS ON ASTRONAUTICS}

$\mathrm{T}$ HE Third International Congress on AstroSeptember 1-6, was attended by fifty official delegates and more than a hundred visitors. During the first two days the business of the International Astronautical Federation was discussed. The major item was the constitution of the Federation, which was agreed to and signed by all societies represented, these being the national societies of the Argentine, Austria, Denmark, Germany, Great Britain, Holland, Italy, Norway, Sweden and Switzerland, together with the American Rocket Society, Chicago Rocket Society, Detroit Rocket Society, Pacific Rocket Society and Reaction Research Society of the United States. The following officers were elected : President, Dr. E. Sänger (Germany) ; Vice-Presidents, Andrew G. Haley (United States) and Dr. L. R. Shepherd (Great Britain) ; Honorary Vice-President for International Relations, Dr. G. Loeser (Germany); and Secretary, J. Stemmer (Switzerland).

Of the twenty-three papers presented during the technical sessions the majority were devoted to problems of detail in space-flight. In his paper "Interorbital Transfer with Minimum Propellant Exponditure", D. F. Lawden (British Interplanetary Society) discussed the problem of the transfer of a rocket between two coplanar elliptic orbits, about the same centre of inverse square law attraction, along a trajectory requiring a minimum of propellant expenditure. His solution involved a number of impulsive thrusts, and equations were obtained from which the magnitude and directions of these thrusts could be calculated in particular cases. In the case of transfer between circular orbits, the solution was shown to be equivalent to that due to Hohmann'.

In "Exposure Hazards from Cosmic Radiation at Extreme Altitudes and in Free Space", Dr. H. J. Schaefer (American Rocket Society) reviewed the present position. In recent years direct evidence has been obtained of the existence of a component of primary cosmic radiation consisting of heavy nuclei. It is known that other types of primary radiation and secondary radiation are not dangerous, but this discovery places the problem of a possible exposure hazard for human beings, in the region of the primary radiation, on a new basis. The heavy primaries are characterized by an extremely high specific ionization and by a large radial spread of the ionization dose around the particle tracks. Either of these properties might endow them with a greatly increased biological effectiveness which cannot be measured effectively in terms of ordinary biological dosage units. The screening effect of the geomagnetic field is such, however, that these particles of comparatively low energy and heavy nuclei would be almost totally excluded from reaching the earth except near the poles. It remains an open question whether such particles are present or not in large quantities in the primary cosmic-ray spectrum in free space away from a body with a shielding magnetic field. It appears that it would probably be safe for human beings to venture a few hundred miles from the earth's surface, but no definite statement can be made for more remote space. A further alternative is the possible existence of a heliomagnetic field which could screen part of the solar system. However, no clear experimental evidence of its existence is available.

Experiments to investigate the biological effects of these heavy primaries were described by Prof. J. Eugster (Schweizerische Astronautische Arbeitsgemeinschaft) in his paper "Die biologische Wirkung der Kosmischen Strahlung, Methoden und neueste Ergebnisse". Investigation of the biological effects on eggs of Artemia salina and human and animal living tissue was carried out by taking the specimens to heights of $28,000-30,000 \mathrm{~m}$. in sounding-balloons. Direct hits by highly ionizing components on Artemia eggs had a lethal effect, the hatching-rate after exposure being zero. The comparative rates at the surface and at a station shielded by some $2,100 \mathrm{~m}$. of rock were 96 and 9 per cent, respectively. The low hatching-rate of the eggs exposed underground was due to considerable neutron radiation which was found to be present. Evaluation of the results of exposure of human and animal tissues was not complete.

A further medical problem of great interest in astronautics was discussed by Dr. A. E. Slater (British Interplanetary Society) in his paper "Sensory Perceptions of the Weightless Condition". It has been suggested that the otolith organs of the inner ear might give confused results when not subjected to a gravitational force or acceleration, and might thus induce a feeling of 'space-sickness'-perhaps akin to sea-sickness. In the weightless condition, the impulse frequency of the maculæ might or might not fall to zero. If there is a certain basic minimum frequency which is present during the weightless 